\title{
An Uncertainty Estimation Framework for Probabilistic Object Detection
}

\author{
Zongyao Lyu, Nolan B. Gutierrez, and William J. Beksi
}

\begin{abstract}
In this paper, we introduce a new technique that combines two popular methods to estimate uncertainty in object detection. Quantifying uncertainty is critical in realworld robotic applications. Traditional detection models can be ambiguous even when they provide a high-probability output. Robot actions based on high-confidence, yet unreliable predictions, may result in serious repercussions. Our framework employs deep ensembles and Monte Carlo dropout for approximating predictive uncertainty, and it improves upon the uncertainty estimation quality of the baseline method. The proposed approach is evaluated on publicly available synthetic image datasets captured from sequences of video.
\end{abstract}

\section{INTRODUCTION}

Object detection is a central task in computer vision and a necessary capability for many robotic operations. Models for object detection have achieved high mean average precision (mAP) on datasets such as ImageNet [1], the PASCAL Visual Object Classes [2], [3], and the Microsoft Common Objects in Context (COCO) [4]. However, these models can fail when evaluated in dynamic scenarios which commonly contain objects from outside the training dataset. The mAP metric encourages detectors to output many detections for each image, yet it does not provide a consistent measure of confidence other than a higher score indicating that the system is more accurate. Nonetheless, robotic vision systems must be able to cope with diverse operating conditions such as variations in illumination and occlusions in the surrounding environment.

The ACRV Robotic Vision Challenge on Probabilistic Object Detection (PrOD) [5] introduces a variation on conventional object detection tasks, which requires quantification of spatial and semantic uncertainty with the use of a new evaluation measure called the probability-based detection quality (PDQ) [6]. Different from mAP, the PDQ jointly evaluates the quality of spatial and label uncertainty, foreground and background separation, and the number of true positive (correct), false positive (spurious), and false negative (missed) detections. In addition, the datasets provided by the challenge are video sequences comprising various scenes that have been generated from high-fidelity simulations.

In this work, we extend our initial groundwork [7] for addressing the issue of uncertainty estimation in object detection (Fig. 1). This includes the exploration of two popular uncertainty estimation techniques: deep ensembles [8] and Monte Carlo (MC) dropout [9]. We apply these techniques jointly on two detectors, Hybrid Task Cascade [10] and Grid

Z. Lyu, N.B. Gutierrez, and W.J. Beksi are with the Department of Computer Science and Engineering, University of Texas at Arlington, Arlington, TX, USA. Emails: zongyao.lyu@mavs.uta.edu, nolan.gutierrez@mavs.uta.edu, william.beksi@uta.edu

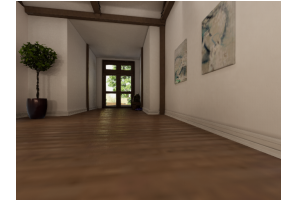

(a)

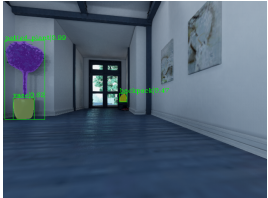

(b)

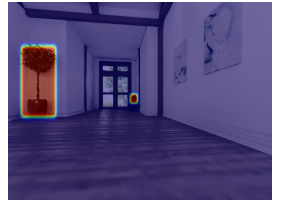

(c)
Fig. 1. An example of a raw input image (a), object detections (b), and the corresponding heatmaps associated with the bounding boxes (c). Our object detection system represents the location of objects as probabilistic bounding boxes. This allows a detector to express spatial uncertainty by inducing a probability distribution over the pixels of the object.

R-CNN [11], to produce probabilistic bounding boxes which allow a detector to express spatial uncertainty. Furthermore, semantic (i.e., label) uncertainty is quantified by a probability distribution over the known classes for each detection. In summary, our contributions are the following.

- We devise a novel aggregation of two prominent techniques to quantify uncertainty in object detection models.

- We create a new heuristic for improving false positive label quality.

- We provide an easy-to-implement method for merging ensembles of detections.

- We introduce high-speed tuning of the hyperparameters for increasing PDQ scores.

- We show that non-maximum suppression (NMS) can be used as a post-processing step to keep all detections.

Our source code is available at [12].

The remainder of this paper is organized as follows. We describe related work in Section II. Section III provides the background information for calculating the PDQ score. Our framework for probabilistic object detection is presented in Section IV. In Section V, we report our experimental results on the validation data provided by the PrOD challenge. We conclude in Section VI with a discussion of future work.

\section{RELATED WORK}

\section{A. Object Detection}

The goal of object detection is to localize and recognize instances of particular categories of objects within an image. Current state-of-the-art object detection systems utilize the power of deep neural networks (DNNs) [13] to output a softmax score indicating the class label and a bounding box denoting the location of each object of a known class. DNN-based object detectors can be categorized into onestage detection models such as YOLO [14] and its variations [15], [16], [17], [18], and two-stage detection models such as R-CNN [19] and its variants [20], [21], [22], [23]. Both categories have advantages over each other. 
Two-stage detectors include a region proposal generator that produces a set of proposals and extracts features from each proposal in the first stage, followed by a second stage of classification and bounding-box regression. Conversely, onestage detectors conduct the prediction of object classes and bounding boxes directly on the image feature maps without a region proposal generation step. Two-stage detectors generally achieve higher accuracy, but they have a relatively slow detection speed. On the other hand, one-stage detectors are more time efficient and have greater applicability to real-time detection, nevertheless they exhibit lower accuracy.

Although the performance of object detectors has been greatly improved with the recent development of deep learning algorithms, current state-of-the-art DNN-based detectors may give high classification probabilities to falsely detected objects. This occurs due to the fact that they output deterministic predictions of bounding boxes without information regarding how certain the predicted results are. Thus, DNN detectors can be unreliable in practical applications and relying on softmax scores and predicted boxes alone is not sufficient.

\section{B. Bayesian Neural Networks for Uncertainty Estimation}

To cope with these limitations, one may extend the classic neural network to produce additional data which can be adapted to supplement the following values: label and spatial uncertainty. This information can then be used to augment the classification score and regressed bounding boxes, thus producing more reliable predictions. Traditionally, Bayesian methods automatically infer hyperparameters by marginalizing them out of the posterior distribution. They can naturally express uncertainty in parameter estimates and propagate it to the predicted results.

Bayesian neural networks (BNNs) [24], [25], [26] offer a natural way to integrate Bayesian modeling with DNNs. BNNs provide a mathematically grounded foundation to reason about uncertainty in DNNs by learning a prior distribution over the weights of a neural network. Not only is each weight in a BNN a random variable, but also the output of the network is a random variable thereby providing a measure of uncertainty. However, compared to non-Bayesian neural networks, BNNs are often computationally intractable which makes them hard to apply in practice.

\section{Dropout as a Bayesian Approximation}

Dropout [27], [28] was first proposed as a technique for training neural networks by randomly omitting neuron units during training to prevent overfitting. It helps prevent co-adaptations whereby a unit relies on several other specific hidden units being present. Dropout also provides a way of approximately combining many disparate neural network models efficiently. Maeda et al. [29] provide an explanation of dropout from a Bayesian standpoint. From this perspective, we can view dropout as a way to deal with the model selection problem by Bayesian model averaging where each model is weighted in accordance with the posterior distribution.
Gal et al. [30], [9] extend the idea of dropout as approximate Bayesian inference in deep Gaussian processes. They show that dropout can be used at test time to impose a Bernoulli distribution over the network's weights, thus requiring no additional model parameters. They refer to this technique as MC dropout. Following this idea, Miller et al. [31], [32] estimate spatial and semantic uncertainties of detections in open-set conditions as a rejection criteria using MC dropout. Kraus et al. [33] incorporate the methods provided by [30] into an object detector to estimate uncertainty, while improving the detection performance of the baseline approach.

\section{Uncertainty Estimation using Deep Ensembles}

Ensembles are one way of improving accuracy in object detection by incorporating detection models. The basic idea is that detectors with contrasting network structures have a different detection performance for distinct objects in an image. As a result, the accuracy and robustness of the detector predictions can be improved due to the application of ensemble methods. For example, Lakshminarayanan et al. [8] propose a non-Bayesian alternative to BNNs by training an ensemble of multiple networks independently. When combined together, the networks behave differently for given inputs hence expressing uncertainty.

\section{E. Probabilistic Object Detection}

As opposed to assuming constant uncertainty, a detection system can assess its own quality by estimating the ambiguity of its task performance. Kendall and Gal [34] identify evaluating spatial and semantic uncertainties as paramount importance, especially in applications where classifications need to be safely clarified. Sirinukunwattana et al. [35] regress probability values for the classification of nuclei in routine colon cancer histology images. Tanno et al. [36] apply Bayesian image quality transfer to dMRI super-resolution by applying sub-pixel convolutions to $3 \mathrm{D}$ tractography. In addition, the authors provide uncertainty maps on a pixelwise basis. A method for improving detection accuracy by modeling bounding boxes with Gaussian parameters is put forth by Choi et al. [37]. Wang et al. [38] propose optimizing PDQ scores by focusing on training-time augmentations. A key contribution of their work was the insight that a system could become more certain of its predictions by feeding in gamma corrected images during training time. Ammirato and Berg [39] examine how a system designed for mAP, specifically Mask-RCNN [22], performs in an environment that evaluates the PDQ metric.

In contrast to the aforementioned works, we combine MC dropout and deep ensembles, and apply it to the object detection problem. We show that the uncertainty estimation performance can be improved over simply using deep ensembles. In addition, whereas previous approaches merged observations by averaging, our approach takes the merged box to be the box for which the system is more confident. When dealing with ensembles, this approach makes sense since object detectors are often distinctly confident with 
predictions of classes and objects with certain characteristics such as appearance or frequency in the dataset.

\section{BACKGROUND}

Consider a ground-truth object $G$ and a detection $D$. Associated with $G$ is a segmentation mask $S$ and a label $c$, along with a bounding box $B$. The segmentation mask $S$ corresponds to the true bounding box and determines which pixels belong to $G$, while $B$ is the set of pixels within the bounding box associated with the object. $D$ consists of a probability distribution over a label vector $\boldsymbol{l}$, a segmentation mask $\hat{S}$, and a probability that some pixel belongs to the segmentation mask $P(\boldsymbol{x} \in \hat{S}) . \hat{S}$ coincides to the pixels within the detection's predicted bounding box. The spatial quality $Q_{\hat{S}}(G, D)$ of this detection is defined by

$\exp \left(\frac{1}{N} \sum_{\boldsymbol{x} \in S} \log (P(\boldsymbol{x} \in \hat{S}))+\frac{1}{N} \sum_{\boldsymbol{x} \in\{S-B\}} \log (1-P(\boldsymbol{x} \in \hat{S}))\right)$,

where $N$ is the number of pixels in the true bounding box $S$, and the two terms in the exponent are known as the foreground and background losses, respectively. $Q_{S}$ is one if the pixels in the ground-truth segmentation are each given probability one, and all the remaining pixels in the set $S-B$ are assigned probability zero. Next, we can calculate the label quality $Q_{l}(G, D)$ by

$$
Q_{L}(G, D)=\boldsymbol{l}_{c}
$$

where $\boldsymbol{l}_{c}$ is the probability of the detection belonging to class $c$ as predicted by the object detector. The pairwise probabilistic detection quality score (pPDQ) can then be calculated by

$$
\operatorname{pPDQ}(G, D)=\sqrt{Q_{\hat{S}} \cdot Q_{L}} .
$$

Let $\boldsymbol{q}_{i j}$ represent the pPDQ of the $j$ th detection in the $i$ th frame. Then the overall PDQ score can be found by

$$
\operatorname{PDQ}(G, D)=\frac{1}{\sum_{i=1}^{F}\left(\mathrm{TP}_{i}+\mathrm{FN}_{i}+\mathrm{FP}_{i}\right)} \sum_{i=1}^{F} \sum_{j=1}^{\mathrm{TP}_{i}} \boldsymbol{q}_{i j},
$$

where $F$ constitutes the number of frames, and $\mathrm{TP}_{i}, \mathrm{FN}_{i}, \mathrm{FP}_{i}$ represent the number of true positives, false negatives, and false positives in the $i$ th frame, respectively. The PDQ measure allows for uncertainty to be modeled in the location of an object which is represented as a probabilistic bounding box. In particular, the corners of the bounding box are 2D Gaussians and a covariance matrix is given for each corner to express a spatial uncertainty over the pixels. The label uncertainty is described as a full probability distribution over the known classes for each detection.

\section{Probabilistic Object Detection Framework}

We detail the key components of our framework for probabilistic object detection in this section. To begin, we describe the datasets and data preprocessing steps. Next, we introduce the detection models and explain how our system utilizes deep ensembles with $\mathrm{MC}$ dropout for uncertainty estimation.
This is followed by a discussion of the heuristics, merging methods, and post-processing techniques we implemented to improve the PDQ scores. An overview of our system is shown in Fig. 2.

\section{A. Datasets and Data Preprocessing}

The video sequences provided by the PrOD challenge are divided into test, test-dev, and validation sets. The validation set contains the released ground truth and is used to evaluate our framework. It consists of around 21500 images with 56580 ground-truth objects in total. The 30 classes of the validation dataset are a subset of 80 classes annotated from COCO. We use models pretrained on COCO and filter out the 50 classes that are not evaluated by the challenge.

\section{B. Detection Models}

We employ two recent well-performing detection models for the task of first-stage detection. The first model is GridRCNN [11], which applies a grid-guided mechanism in place of the traditional box offset regression, for high-quality localization. The second model is Hybrid Task Cascade (HTC) [10], an effective cascade architecture for object detection and instance segmentation.

\section{Combining Deep Ensembles with Monte Carlo Dropout}

For each detector, we retain dropout layers in the model during test time and then make an ensemble of the two models. Our approach is similar to NMS [40]. We simplify and improve the method proposed by Miller et al. [31] in which detections are grouped into observations by IoU. A key difference in our approach is we take the merged bounding box from each group to be the box that the model is most confident about (where confidence is measured by the highest label score). To merge predictions, we group detections by IoU via NMS, and we keep the most confident detections from each observation.

First, NMS partitions boxes $B$ into groups $G$ such that any two boxes in a group $G_{i}$ has an IoU above a threshold $\lambda$ between 0 and 1. Each group must be sorted by confidence scores. The list of boxes contains the top-left and bottomright corners, and the confidence of each box's label. Next, each of these observations represented as groups of bounding boxes are reduced to the most confident bounding box and label. Finally, the covariance matrices of every detection are computed. The procedure for merging the detections from our deep ensemble is shown in Algorithm 1.

\section{Heuristics for Improving the PDQ Score}

We can maximize the PDQ score by optimizing metrics that evaluate the quality of object detections. As an example, we may increase the PDQ score by reducing the number of objects in a detection that are incorrectly classified [39]. Alternatively, researchers have demonstrated that inflating label scores to one increases the average label quality and thus the PDQ score. To systematically find the optimal parameters, we save the boxes that are obtained from each configuration of the model and uniformly search the hyperparameter space. 

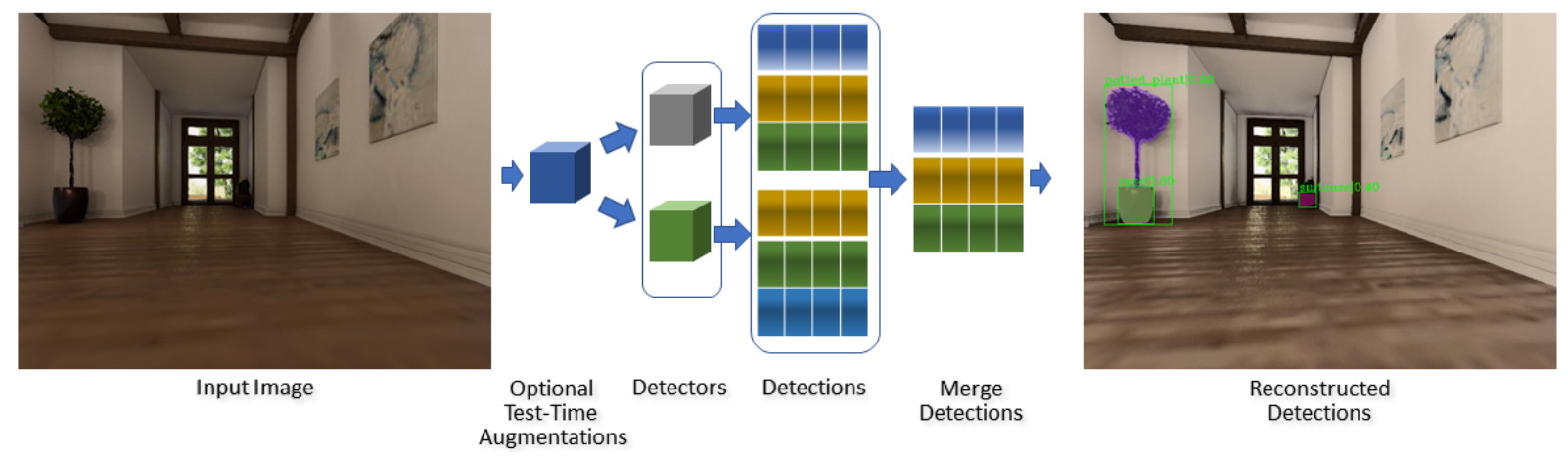

Fig. 2. An overview of our deep ensemble architecture for probabilistic object detection.

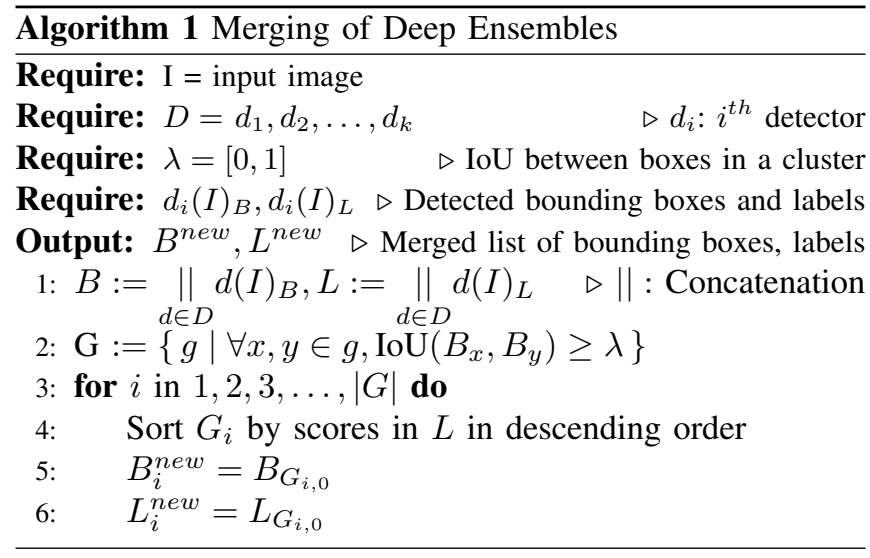

In the following paragraphs, we describe the details for each heuristic employed to improve the PDQ score.

Threshold Filtering: Rejection of bounding boxes that have low-confidence has the greatest impact on localization performance and the PDQ score. Succinctly, the number of false negatives, false positives, and true positives is affected, therefore producing a wide range of PDQ scores. While other work on this topic has found an optimal confidence threshold of 0.5 [39], we've discovered that higher thresholds significantly increase the number of false negatives and thus reduce the PDQ score. In our search of the choice hyperparameters, we've found that we can set the confidence threshold as low as possible but only when running NMS once more with a low IoU threshold.

Bounding Box Reduction: The PDQ score can be substantially lowered by confidently labeling pixels in the background. To lessen this issue, we center crop the bounding box whereby the box's width and height are reduced by a specified proportion. The PDQ score increases by reducing the ratio of background pixels to foreground pixels. We specify our reduction ratio to be 0.1 thereby gaining better results.

Improving False Positive Quality: False positive label quality is lower when incorrectly classified objects assign low probabilities to correct labels. We conceive a novel heuristic by noting that uniformly distributing probabilities over the rest of the labels in the label vector $l$ can maximize this metric over all testing data. Concretely,

$$
l_{i}= \begin{cases}S, & l_{i} \text { corresponds to the detection label, } \\ \frac{1-S}{30}, & \text { otherwise }\end{cases}
$$

where $S$ is the score of the bounding box's label and 30 is the number of classes.

Covariance Matrix Calculation: We experimented with traditional covariance calculations and fixed covariances. Notwithstanding, we improve our results by setting the diagonal of the covariance matrix, which represents the spatial uncertainty, proportional to the bounding box's width and height [39]. As an example, if we use a proportion of 0.10 for a 50 by 60 bounding box, then the covariance matrix would be defined as $[[5,0],[0,6]]$ for both the top-left and bottom-right corners.

Non-Maximal Suppression: Contemporary work has shown that the PDQ score can be improved by removing low-confidence detections. We've verified that this heuristic does improve the score, nevertheless this result can be outperformed by adding an additional NMS post-processing step. However, allowing the evaluation of low-confidence boxes may increase the number of false positives. To avoid this situation, we allow larger higher-confident boxes to absorb smaller lower-confident boxes which reduces the number of false positives.

\section{EXPERIMENTAL RESULTS}

This section presents our experimental results on the validation data provided by the PrOD challenge. Our implementation is based on MMDetection [41], an open-source object detection toolbox. The experiments were performed on a Ubuntu 18.04 machine with an Intel Core i7-8700 CPU, 32GB of RAM, and an NVIDIA Quadro P4000 GPU.

Both detectors used in the experiments are models pretrained on the COCO dataset with the ResNeXt101 backbone. The results of using an ensemble model composed of HTC and Grid R-CNN with various threshold parameters are shown in Table I. In our experiments, lower threshold values provided higher scores. Table II shows the effect of reducing the bounding box size of all detections on the PDQ scores. Here we found that reduction of the bounding box size by $10 \%$ results in better performance. Table III shows 
how the PDQ score changes when scaling the covariance to a different percentage of the box size. We experimented with covariance matrices dependent on the widths and heights of the bounding boxes. The effectiveness of this technique was demonstrated in [39], and our final results applied this heuristic. After exploring various values in our implementation, we found that setting the top-left and bottom-right elements of the covariance matrix equal to a scale of $30 \%$ of the bounding box size gave the best results. We report these findings with different IoU threshold values in Table IV.

Table $\mathrm{V}$ shows the efficacy of the individual HTC and Grid R-CNN detectors, and their ensembles. We observed that a single HTC detector outperforms an ensemble of HTC and Grid R-CNN in our experiments. This indicates that a careful selection of integrated detection models and parameter tuning are necessary to bring about an effective ensemble model, thus further exploration is required. We examined $\mathrm{MC}$ dropout as the uncertainty estimation technique on the HTC and Grid R-CNN detectors individually, and also their ensembles. In practice, this method is equivalent to performing several stochastic forward passes through the network and then taking an average of the results. We chose to sample three passes by adding a dropout rate of 0.3 to the second shared fully-convolutional layer of HTC's region of interest head. After testing a large number of postprocessing parameters, we found that models using dropout consistently demonstrated better quality when compared to models without dropout. The result of an ensemble model with dropout outperforming an ensemble without dropout demonstrates the effectiveness of our idea for integrating these two approaches. The results of the models with dropout are displayed in Table V.

Finally, we compared our most confident box merging (MCBM) strategy with averaging in Table VI. With all other parameters being the same, we present evidence that even when boxes in an observation must have the same label, the detection quality is best whenever we take the merged box to be the most confident box in the observation. Our hypothesis is we should allow the detection system that is most confident about its result have its prediction take over the observation. As expected, the MCBM method achieved the same results with and without the constraint that the boxes in an observation must have the same label.

\section{CONClusion And Future Work}

We presented an uncertainty estimation framework which makes use of the recently introduced PDQ evaluation metric and achieves a top score on the PrOD challenge validation dataset. By extensively exploring the hyperparameter space, we showed the quality of object detections can be maintained by introducing additional post-processing techniques. Likewise, we've shown that post-processing methods designed to increase detection quality may influence one another and the optimization of these techniques can be done offline. We highlighted that the IoU parameter of NMS designed for mAP does not lead to high-quality detections. In fact, we experimentally demonstrated that very low IoU thresholds group more detections together and reduce the number of false positives.

Our forthcoming work in probabilistic object detection will explore the incorporation of manual heuristics into an end-to-end model. For example, it is advantageous to add bias for smaller bounding boxes. Moreover, post-processing steps must be adaptive, i.e., the hyperparameters for these steps may be image dependent. This warrants further investigation into the adequacy of applying computationally intensive methods that occasionally decrease the quality of the performance. More importantly, when applying these methods one should clearly select detectors based on their strengths and weaknesses while being aware of any potential increase in computation.

\section{REFERENCES}

[1] O. Russakovsky, J. Deng, H. Su, J. Krause, S. Satheesh, S. Ma, Z. Huang, A. Karpathy, A. Khosla, M. Bernstein et al., "Imagenet large scale visual recognition challenge," International Journal of Computer Vision, vol. 115, no. 3, pp. 211-252, 2015.

[2] M. Everingham, L. Van Gool, C. K. Williams, J. Winn, and A. Zisserman, "The pascal visual object classes (voc) challenge," International Journal of Computer Vision, vol. 88, no. 2, pp. 303-338, 2010.

[3] M. Everingham, S. A. Eslami, L. Van Gool, C. K. Williams, J. Winn, and A. Zisserman, "The pascal visual object classes challenge: A retrospective," International Journal of Computer Vision, vol. 111, no. 1, pp. 98-136, 2015.

[4] T.-Y. Lin, M. Maire, S. Belongie, J. Hays, P. Perona, D. Ramanan, P. Dollár, and C. L. Zitnick, "Microsoft coco: Common objects in context," in Proceedings of the European Conference on Computer Vision (ECCV). Springer, 2014, pp. 740-755.

[5] J. Skinner, D. Hall, H. Zhang, F. Dayoub, and N. Sünderhauf, "The probabilistic object detection challenge," arXiv preprint arXiv:1903.07840, 2019.

[6] D. Hall, F. Dayoub, J. Skinner, H. Zhang, D. Miller, P. Corke, G. Carneiro, A. Angelova, and N. Sünderhauf, "Probabilistic object detection: definition and evaluation," in Proceedings of the IEEE Winter Conference on Applications of Computer Vision (WACV), 2020, pp. 1031-1040.

[7] Z. Lyu, N. Gutierrez, A. Rajguru, and W. J. Beksi, "Probabilistic object detection via deep ensembles," in Proceedings of the European Conference on Computer Vision (ECCV) Workshops. Springer, 2020, pp. 67-75.

[8] B. Lakshminarayanan, A. Pritzel, and C. Blundell, "Simple and scalable predictive uncertainty estimation using deep ensembles," in Proceedings of the Advances in Neural Information Processing Systems (NeurIPS), 2017, pp. 6402-6413.

[9] Y. Gal and Z. Ghahramani, "Dropout as a bayesian approximation: Representing model uncertainty in deep learning," in Proceedings of the International Conference on Machine Learning (ICML), 2016, pp. 1050-1059.

[10] K. Chen, J. Pang, J. Wang, Y. Xiong, X. Li, S. Sun, W. Feng, Z. Liu, J. Shi, W. Ouyang et al., "Hybrid task cascade for instance segmentation," in Proceedings of the IEEE Conference on Computer Vision and Pattern Recognition (CVPR), 2019, pp. 4974-4983.

[11] X. Lu, B. Li, Y. Yue, Q. Li, and J. Yan, "Grid r-cnn," in Proceedings of the IEEE Conference on Computer Vision and Pattern Recognition (CVPR), 2019, pp. 7363-7372.

[12] https://github.com/robotic-vision-lab/Deep-Ensembles-ForProbabilistic-Object-Detection.

[13] C. Szegedy, A. Toshev, and D. Erhan, "Deep neural networks for object detection," in Proceedings of the Advances in Neural Information Processing Systems (NeurIPS), 2013, pp. 2553-2561.

[14] J. Redmon, S. Divvala, R. Girshick, and A. Farhadi, "You only look once: Unified, real-time object detection," in Proceedings of the IEEE Conference on Computer Vision and Pattern Recognition (CVPR), 2016, pp. 779-788.

[15] W. Liu, D. Anguelov, D. Erhan, C. Szegedy, S. Reed, C.-Y. Fu, and A. C. Berg, "Ssd: Single shot multibox detector," in Proceedings of the European Conference on Computer Vision (ECCV). Springer, 2016, pp. 21-37. 


\begin{tabular}{|c|c|c|c|c|c|c|c|c|}
\hline Threshold & PDQ Score & Avg. pPDQ & Avg. FP & Avg. SQ & Avg. LQ & TPs & FPs & FNs \\
\hline \hline $\mathbf{0 . 0 1 8}$ & $\mathbf{2 2 . 5 6 9}$ & 0.409 & $\mathbf{0 . 8 3 5}$ & 0.464 & 0.498 & $\mathbf{5 8 9 6 1}$ & 112689 & $\mathbf{2 9 1 2 8}$ \\
\hline 0.05 & 22.534 & 0.446 & 0.747 & 0.483 & 0.551 & 53036 & 66395 & 35053 \\
\hline 0.1 & 22.468 & 0.481 & 0.648 & 0.498 & 0.602 & 48176 & 42652 & 39913 \\
\hline 0.3 & 22.006 & 0.544 & 0.424 & 0.520 & 0.703 & 39940 & 18615 & 48149 \\
\hline 0.5 & 20.919 & $\mathbf{0 . 5 9 1}$ & 0.270 & $\mathbf{0 . 5 3 7}$ & $\mathbf{0 . 7 8 0}$ & 33809 & $\mathbf{1 0 2 7 7}$ & 54280 \\
\hline
\end{tabular}

TABLE I

THE RESULTS OF AN ENSEMBLE MODEL USING DIFFERENT THRESHOLD VALUES ON THE VALIDATION DATASET (AVG. SQ=AVERAGE SPATIAL QUALITY, AVG. LQ=AVERAGE LABEL QUALITY, TPS=TRUE POSITIVES, FPS=FALSE POSITIVES, FNS=FALSE NEGATIVES).

\begin{tabular}{|c|c|c|c|c|c|c|c|c|}
\hline Box Ratio & PDQ Score & Avg. pPDQ & Avg. FP & Avg. SQ & Avg. LQ & TPs & FPs & FNs \\
\hline \hline 0.05 & 22.084 & 0.399 & 0.835 & 0.445 & 0.497 & $\mathbf{5 9 0 2 6}$ & 112799 & $\mathbf{2 9 0 6 3}$ \\
\hline $\mathbf{0 . 1}$ & $\mathbf{2 2 . 5 6 9}$ & $\mathbf{0 . 4 0 9}$ & $\mathbf{0 . 8 3 5}$ & $\mathbf{0 . 4 6 4}$ & 0.498 & 58961 & 112689 & 29128 \\
\hline 0.2 & 19.861 & 0.360 & 0.835 & 0.383 & 0.499 & 58848 & $\mathbf{1 1 2 4 1 4}$ & 29241 \\
\hline 0.3 & 12.882 & 0.235 & 0.834 & 0.219 & $\mathbf{0 . 5 0 0}$ & 58509 & 112471 & 29580 \\
\hline
\end{tabular}

TABLE II

THE RESULTS OF AN ENSEMBLE MODEL USING DIFFERENT BOUNDING BOX RATIOS ON THE VALIDATION DATASET (AVG. SQ=AVERAGE SPATIAL

QUALITY, AVG. LQ=AVERAGE LABEL QUALITY, TPS=TRUE POSITIVES, FPS=FALSE POSITIVES, FNS=FALSE NEGATIVES ).

\begin{tabular}{|c|c|c|c|c|c|c|c|c|}
\hline Covariance Scale & PDQ Score & Avg. pPDQ & Avg. FP & Avg. SQ & Avg. LQ & TPs & FPs & FNs \\
\hline \hline 0.1 & 21.894 & 0.402 & 0.834 & 0.462 & $\mathbf{0 . 5 0 2}$ & 58275 & 113517 & 29814 \\
\hline 0.2 & 22.536 & $\mathbf{0 . 4 1 1}$ & $\mathbf{0 . 8 3 5}$ & $\mathbf{0 . 4 7 3}$ & 0.500 & 58605 & 113146 & 29484 \\
\hline $\mathbf{0 . 3}$ & $\mathbf{2 2 . 5 6 9}$ & 0.409 & 0.835 & 0.464 & 0.498 & 58961 & 112689 & 29128 \\
\hline 0.4 & 22.458 & 0.403 & 0.835 & 0.450 & 0.495 & 59421 & 112020 & 28668 \\
\hline 0.5 & 22.283 & 0.397 & 0.835 & 0.437 & 0.492 & $\mathbf{5 9 7 3 7}$ & $\mathbf{1 1 1 5 5 7}$ & $\mathbf{2 8 3 5 2}$ \\
\hline
\end{tabular}

TABLE III

THE RESULTS OF AN ENSEMBLE MODEL USING DIFFERENT COVARIANCE SCALES ON THE VALIDATION DATASET (AVG. SQ=AVERAGE SPATIAL QUALITY, AVG. LQ=AVERAGE LABEL QUALITY, TPS=TRUE POSITIVES, FPS=FALSE POSITIVES, FNS=FALSE NEGATIVES).

\begin{tabular}{|c|c|c|c|c|c|c|c|c|}
\hline IoU Threshold & PDQ Score & Avg. pPDQ & Avg. FP & Avg. SQ & Avg. LQ & TPs & FPs & FNs \\
\hline 0.1 & 21.966 & $\mathbf{0 . 4 1 9}$ & 0.817 & 0.468 & $\mathbf{0 . 5 1 4}$ & 54194 & $\mathbf{8 4 0 3 6}$ & 33895 \\
\hline 0.2 & 22.395 & 0.411 & 0.827 & 0.465 & 0.504 & 57398 & 100192 & 30691 \\
\hline $\mathbf{0 . 3}$ & $\mathbf{2 2 . 5 6 9}$ & 0.409 & 0.835 & 0.464 & 0.498 & 58961 & 112689 & 29128 \\
\hline 0.4 & 22.530 & 0.405 & 0.844 & 0.465 & 0.486 & 60824 & 136205 & 27265 \\
\hline 0.5 & 22.314 & 0.405 & $\mathbf{0 . 8 5 6}$ & $\mathbf{0 . 4 7 2}$ & 0.477 & $\mathbf{6 2 3 3 8}$ & 175034 & $\mathbf{2 5 7 5 1}$ \\
\hline
\end{tabular}

TABLE IV

THE RESULTS OF AN ENSEMBLE MODEL USING DIFFERENT IOU THRESHOLD VALUES ON THE VALIDATION SET (AVG. SQ=AVERAGE SPATIAL QUALITY, AVG. LQ=AVERAGE LABEL QUALITY, TPS=TRUE POSITIVES, FPS=FALSE POSITIVES, FNS=FALSE NEGATIVES ).

\begin{tabular}{|c|c|c|c|c|c|c|c|c|}
\hline Method & PDQ Score & Avg. pPDQ & Avg. FP & Avg. SQ & Avg. LQ & TPs & FPs & FNs \\
\hline HTC & 22.769 & 0.415 & 0.833 & 0.472 & 0.496 & 56054 & 84565 & 32035 \\
\hline HTC + MC dropout & 22.822 & 0.414 & 0.824 & 0.467 & 0.504 & 58498 & 102610 & 29591 \\
\hline Grid R-CNN & 18.014 & 0.410 & 0.799 & 0.465 & 0.514 & 42383 & 42162 & 45706 \\
\hline Grid R-CNN + MC dropout & 18.222 & 0.395 & 0.789 & 0.445 & 0.514 & 48340 & 79423 & 39749 \\
\hline Ensemble & 22.569 & 0.409 & 0.835 & 0.464 & 0.498 & 58961 & 112689 & 29128 \\
\hline Ensemble + MC dropout & 22.599 & 0.417 & 0.818 & 0.469 & 0.515 & 59847 & 122280 & 28242 \\
\hline
\end{tabular}

\section{TABLE V}

THE RESUlTS OF A SINGLE AND AN ENSEMBLE MODEL BEFORE AND AFTER ADDING DROPOUT ON THE VALIDATION DATASET (AVG. SQ=AVERAGE SPATIAL QUALITY, AVG. LQ=AVERAGE LABEL QUALITY, TPS=TRUE POSITIVES, FPS=FALSE POSITIVES, FNS=FALSE NEGATIVES).

\begin{tabular}{|c|c|c|c|c|c|c|c|c|}
\hline Method & PDQ Score & Avg. pPDQ & Avg. FP & Avg. SQ & Avg. LQ & TPs & FPs & FNs \\
\hline Ensemble (averaging) & 12.642 & 0.223 & 0.907 & 0.324 & 0.238 & 54622 & 90024 & 33467 \\
\hline Ensemble (same labels, averaging) & 14.286 & 0.246 & 0.887 & 0.323 & 0.294 & 57425 & 101779 & 30664 \\
\hline Ensemble (ours) & 22.569 & 0.409 & 0.835 & 0.464 & 0.498 & 58961 & 112689 & 29128 \\
\hline
\end{tabular}

TABLE VI

A COMPARISON OF ENSEMBLE MODELS USING AVERAGING AND OUR MERGING STRATEGY ON THE VALIDATION DATASET (AVG. SQ=AVERAGE SPATIAL QUALITY, AVG. LQ=AVERAGE LABEL QUALITY, TPS=TRUE POSITIVES, FPS=FALSE POSITIVES, FNS=FALSE NEGATIVES). 
[16] J. Redmon and A. Farhadi, "Yolo9000: better, faster, stronger," in Proceedings of the IEEE Conference on Computer Vision and Pattern Recognition (CVPR), 2017, pp. 7263-7271.

[17] —, "Yolov3: An incremental improvement," arXiv preprint arXiv:1804.02767, 2018.

[18] A. Bochkovskiy, C.-Y. Wang, and H.-Y. M. Liao, "Yolov4: Optimal speed and accuracy of object detection," arXiv preprint arXiv:2004.10934, 2020.

[19] R. Girshick, J. Donahue, T. Darrell, and J. Malik, "Rich feature hierarchies for accurate object detection and semantic segmentation," in Proceedings of the IEEE Conference on Computer Vision and Pattern Recognition (CVPR), 2014, pp. 580-587.

[20] R. Girshick, "Fast r-cnn," in Proceedings of the IEEE International Conference on Computer Vision (ICCV), 2015, pp. 1440-1448.

[21] S. Ren, K. He, R. Girshick, and J. Sun, "Faster r-cnn: Towards realtime object detection with region proposal networks," in Proceedings of the Advances in Neural Information Processing Systems (NeurIPS), 2015, pp. 91-99.

[22] K. He, G. Gkioxari, P. Dollár, and R. Girshick, "Mask r-cnn," in Proceedings of the IEEE Conference on Computer Vision and Pattern Recognition (CVPR), 2017, pp. 2961-2969.

[23] Z. Cai and N. Vasconcelos, "Cascade r-cnn: Delving into high quality object detection," in Proceedings of the IEEE Conference on Computer Vision and Pattern Recognition (CVPR), 2018, pp. 6154-6162.

[24] J. S. Denker and Y. LeCun, "Transforming neural-net output levels to probability distributions," in Proceedings of the Advances in Neural Information Processing Systems (NeurIPS), 1991, pp. 853-859.

[25] D. J. MacKay, "A practical bayesian framework for backpropagation networks," Neural Computation, vol. 4, no. 3, pp. 448-472, 1992.

[26] J. M. Hernández-Lobato and R. Adams, "Probabilistic backpropagation for scalable learning of bayesian neural networks," in Proceedings of the International Conference on Machine Learning (ICML), 2015, pp. 1861-1869.

[27] G. E. Hinton, N. Srivastava, A. Krizhevsky, I. Sutskever, and R. R. Salakhutdinov, "Improving neural networks by preventing coadaptation of feature detectors," arXiv preprint arXiv:1207.0580, 2012.

[28] N. Srivastava, G. Hinton, A. Krizhevsky, I. Sutskever, and R. Salakhutdinov, "Dropout: a simple way to prevent neural networks from overfitting," Journal of Machine Learning Research, vol. 15, no. 1, pp. 1929-1958, 2014.

[29] S. Maeda, "A bayesian encourages dropout," arXiv preprint arXiv:1412.7003, 2014.

[30] Y. Gal and Z. Ghahramani, "Bayesian convolutional neural networks with bernoulli approximate variational inference," arXiv preprint arXiv:1506.02158, 2015.

[31] D. Miller, L. Nicholson, F. Dayoub, and N. Sünderhauf, "Dropout sampling for robust object detection in open-set conditions," in Proceedings of the IEEE International Conference on Robotics and Automation (ICRA), 2018, pp. 1-7.

[32] D. Miller, F. Dayoub, M. Milford, and N. Sünderhauf, "Evaluating merging strategies for sampling-based uncertainty techniques in object detection," in Proceedings of the IEEE International Conference on Robotics and Automation (ICRA), 2019, pp. 2348-2354.

[33] F. Kraus and K. Dietmayer, "Uncertainty estimation in one-stage object detection," in Proceedings of the IEEE Intelligent Transportation Systems Conference (ITSC), 2019, pp. 53-60.

[34] A. Kendall, Y. Gal, and R. Cipolla, "Multi-task learning using uncertainty to weigh losses for scene geometry and semantics," arXiv preprint arXiv:1705.07115, 2017.

[35] K. Sirinukunwattana, S. E. A. Raza, Y.-W. Tsang, D. R. Snead, I. A. Cree, and N. M. Rajpoot, "Locality sensitive deep learning for detection and classification of nuclei in routine colon cancer histology images," IEEE Transactions on Medical Imaging, vol. 35, no. 5, pp. 1196-1206, 2016.

[36] R. Tanno, D. E. Worrall, A. Ghosh, E. Kaden, S. N. Sotiropoulos, A. Criminisi, and D. C. Alexander, "Bayesian image quality transfer with cnns: exploring uncertainty in dmri super-resolution," in Proceedings of the International Conference on Medical Image Computing and Computer-Assisted Intervention. Springer, 2017, pp. 611-619.

[37] J. Choi, D. Chun, H. Kim, and H. Lee, "Gaussian yolov3: An accurate and fast object detector using localization uncertainty for autonomous driving," Proceedings of the IEEE International Conference on Computer Vision (ICCV), pp. 502-511, 2019.

[38] C.-W. Wang, C.-A. Cheng, C.-J. Cheng, H.-N. Hu, H.-K. Chu, and M. Sun, "Augpod: Augmentation-oriented probabilistic object detec- tion," in Proceedings of the CVPR Workshop on the Robotic Vision Probabilistic Object Detection Challenge, 2019.

[39] P. Ammirato and A. C. Berg, "A mask-rcnn baseline for probabilistic object detection," in Proceedings of the CVPR Workshop on the Robotic Vision Probabilistic Object Detection Challenge, 2019.

[40] D. Li, C. Xu, Y. Liu, and Z. Qin, "Teamgl at acrv robotic vision challenge 1: Probabilistic object detection via staged non-suppression ensembling," in Proceedings of the CVPR Workshop on the Robotic Vision Probabilistic Object Detection Challenge, 2019.

[41] K. Chen, J. Wang, J. Pang, Y. Cao, Y. Xiong, X. Li, S. Sun, W. Feng, Z. Liu, J. Xu et al., "Mmdetection: Open mmlab detection toolbox and benchmark," arXiv preprint arXiv:1906.07155, 2019. 